\title{
Treating a Structural Heart Disease Using a Non-structural Approach: Role of Cardiac Pacing in Hypertrophic Cardiomyopathy
}

\author{
Bernard Benjamin P. Albano, e, Erdie C. Fadreguilan ${ }^{\mathrm{b}}$, Jeffrey M. Chua ${ }^{\mathrm{c}}$, James $\mathrm{Ho}^{\mathrm{c}}$, \\ Ana Beatriz Medrano ${ }^{\mathrm{d}}$
}

\begin{abstract}
Hypertrophic cardiomyopathy (HCM) is the most common genetic cardiovascular disease characterized by a thickened non-dilated ventricle in the absence of another cardiac or systemic condition. Its most important hemodynamic consequence is left ventricular outflow tract (LVOT) obstruction. The primary management strategy of this condition is surgical septal myectomy, but an acceptable alternative treatment in patients who are not suitable for (or who refuse) surgery is alcohol septal ablation (ASA). However, in patients with unfavorable coronary anatomy which precludes ASA (i.e. absence of major septal perforator branch of the left anterior descending (LAD) artery), another reasonable option is dual chamber pacemaker implantation to decrease LVOT outflow gradient. A 77-year-old female, known hypertensive, diabetic with a history of coronary artery disease, presented with 1 -week history of worsening chest pain and shortness of breath. She was admitted as a case of acute coronary syndrome and pneumonia. On workup, 2DED revealed hypertrophic obstructive cardiomyopathy (HOCM) with a demonstrated systolic anterior motion (SAM) of the mitral valve with a peak instantaneous gradient of $194 \mathrm{~mm} \mathrm{Hg}$ across the basal LV cavity. The patient refused surgical myectomy, and ASA was the preferred treatment option. On coronary angiography, there was an incidental finding of absent major septal perforator branch of the LAD coronary artery, rendering her unsuitable for septal ablation. She was referred to electrophysiology for evaluation. She underwent dual chamber pacemaker implantation and documented significant decrease in the peak instantaneous gradient from 194 to $37 \mathrm{~mm} \mathrm{Hg}$, with complete obliteration of SAM and improvement in overall wall motion. She remained stable and asymptomatic after pacemaker insertion until
\end{abstract}

Manuscript accepted for publication December 12, 2016

aDepartment of Adult Cardiology, Philippine Heart Center, East Avenue, Quezon City, Philippines

${ }^{b}$ Division of Electrophysiology, Philippine Heart Center, East Avenue, Quezon City, Philippines

'Division of Invasive Cardiology, Philippine Heart Center, East Avenue, Quezon City, Philippines

${ }^{\mathrm{d}}$ Division of Noninvasive Cardiology, Philippine Heart Center, East Avenue, Quezon City, Philippines

${ }^{\mathrm{e} C}$ Corresponding Author: Bernard Benjamin P. Albano, Department of Adult Cardiology, Philippine Heart Center, East Avenue, Quezon City, Philippines. Email:burn_hp@yahoo.com

doi: https://doi.org/10.14740/cr512w her recent outpatient follow-up (1 year after implantation). We present a case of HCM with congenitally absent major septal perforator branch coronary artery treated with dual chamber pacemaker implantation. To our knowledge, this is the first reported angiographically absent first (major) septal perforator coronary anatomy in the setting of HCM, and also the first description of dual chamber pacemaker implantation to relieve the LVOT obstruction. Although the role of dual chamber pacing has become limited in HCM because surgical myectomy and septal ablation have resulted in better decrease in LV outflow gradient and symptom improvement, this modality remains essential and may still be considered as the treatment strategy-of-choice in patients who are unsuitable for surgical myectomy and ASA.

Keywords: Hypertrophic cardiomyopathy; Hypertrophic obstructive cardiomyopathy; Surgical myectomy; Alcohol septal ablation; Dual chamber pacemaker implantation

\section{Introduction}

Hypertrophic cardiomyopathy (HCM) is the most common genetic cardiovascular disease characterized by a thickened nondilated ventricle in the absence of other cardiac or systemic condition [1]. It is a global disease with a reported prevalence of $0.2 \%$ in the general population [2]. Its clinical diagnosis is made by imaging with two-dimensional echocardiography. The most important hemodynamic consequence of this condition is left ventricular outflow tract (LVOT) obstruction in which $70 \%$ of patients develop dynamic LV outflow gradient of $30 \mathrm{~mm} \mathrm{Hg}$ or greater. In the obstructive form, there is subaortic mechanical impedance to LV outflow, producing markedly increased intraventricular pressures that, over time, may be detrimental to LV function as a result of increased myocardial wall stress and oxygen demand. The most common obstructive mechanism $(90 \%)$ is the systolic anterior motion (SAM) of the mitral valve, and the remaining causes (10\%) include intraventricular obstruction caused by systolic contact of septum with a papillary muscle that is anomalously positioned and may insert directly into anterior mitral leaflet.

In patients with severe drug-refractory NYHA class III and IV symptoms, the preferred primary management is surgical septal myomectomy $[2,3]$. In patients who are not candidates for surgery, an alternative treatment option is alcohol septal 


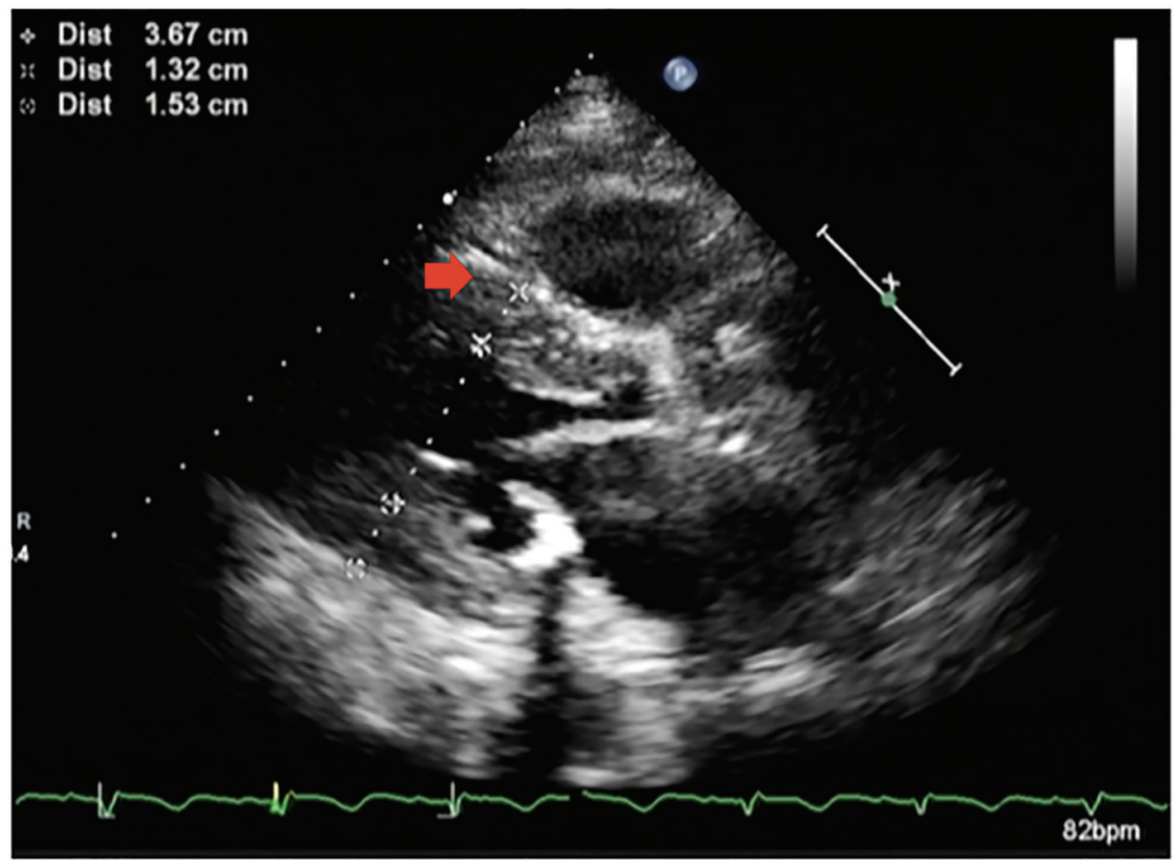

Figure 1. Parasternal long axis echocardiographic image of the patient showing hypertrophied septum (red arrow) and reduced left ventricular cavity size.

ablation (ASA) $[1,4,5]$. This modality involves injection of alcohol into a major septal perforator coronary artery to create necrosis and permanent transmural myocardial infarction localized to the proximal ventricular septum which results in progressive thinning of the LV wall and a resultant decrease in LV outflow gradient [6]. However, in patients with congenitally absent major septal perforator branches of the left anterior descending (LAD) coronary artery, the performance of ASA may be ineffective. Implantation of a dual chamber pacemaker was proposed as an alternate treatment for these patients with severe symptomatic obstructive HCM [7-9]. By pacing the right ventricular apex with maintenance of atrioventricular synchrony,

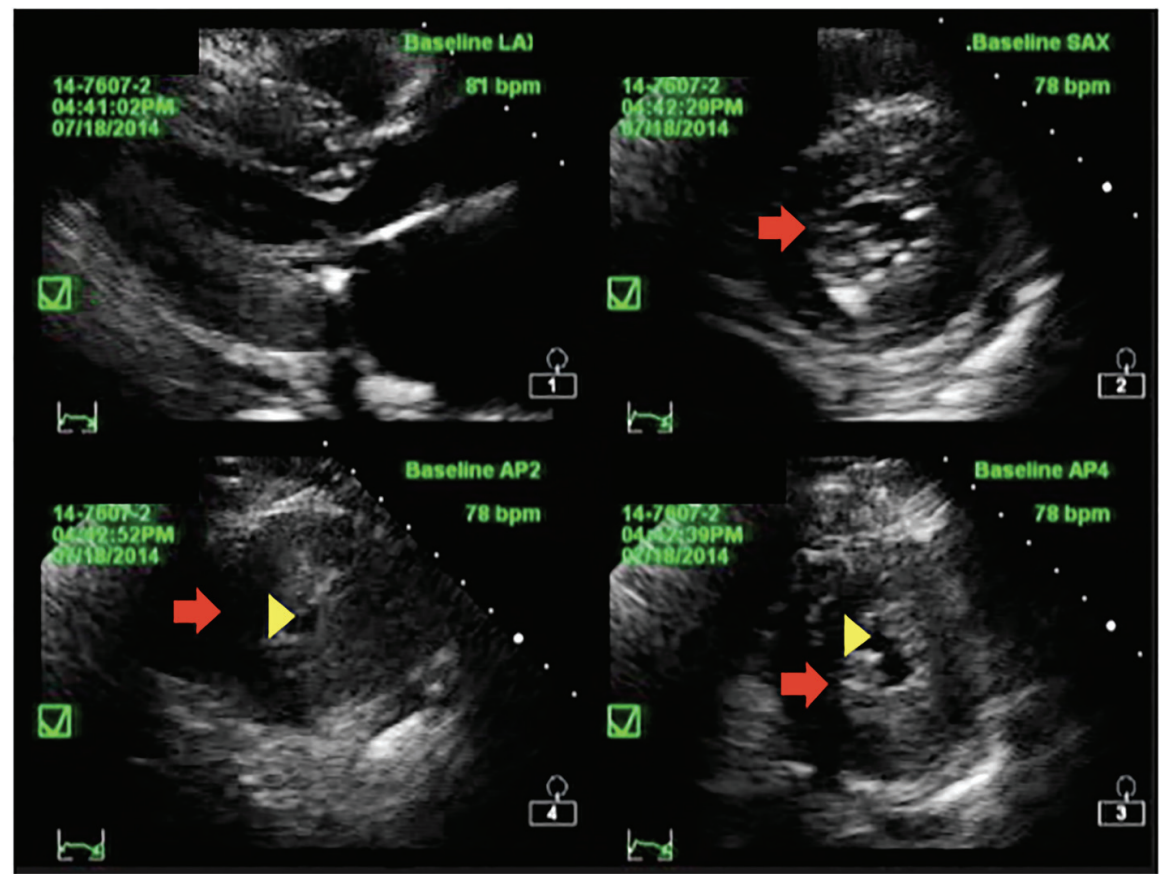

Figure 2. Short axis echocardiographic image of the patient showing concentric hypertrophy of the left ventricular wall (red arrow) and reduced LV cavity (yellow arrowhead). 

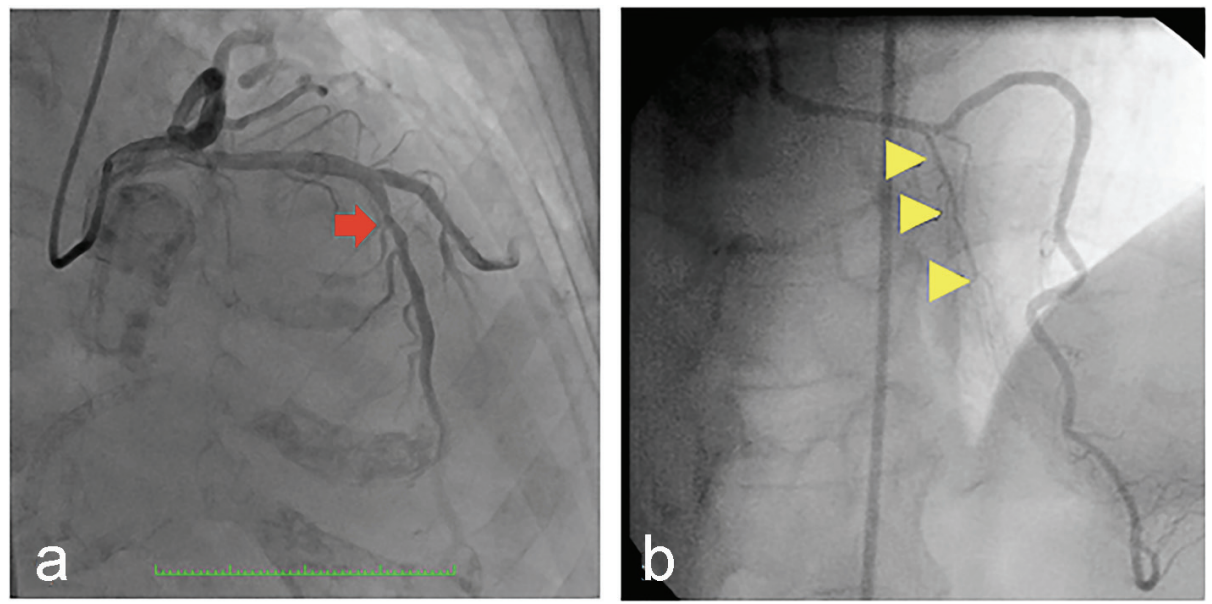

Figure 3. (a) Coronary angiography of the patient showing the left anterior descending artery (arrow) which was a good-sized vessel, with no significant stenosis but with absent first (major) septal perforator branch. (b) Normal major septal perforator (yellow arrowheads) originating from the long LAD from a 52-year-old female [11].

this results in a decrease in the LVOT gradient and improvement of symptoms. Importantly, however, its role in HCM has become limited due to lack of evidence of efficacy [10].

This is a case of a symptomatic HCM in an elderly who refused surgical myectomy, and was planned for ASA but did not push through due to incidental finding of absent major septal perforator branch of the LAD. Due to persistent refusal of surgery, she was referred to electrophysiology and underwent dual chamber pacemaker implantation. There was noted significant improvement of symptoms and she was discharged hemodynamically stable. To our knowledge, this is the first reported case of congenital absence of septal perforator artery in the setting of HCM and also the first description of successful treatment of LVOT gradient using dual chamber pacing in the local setting.

\section{Case Report}

This is a case of EA, a 77-year-old Filipino female, diabetic, hypertensive with a history of coronary artery disease who underwent percutaneous coronary angioplasty of the right coronary artery (RCA) in 2000. One week prior to consult, she complained of occasional chest heaviness aggravated by effort and worsening shortness of breath. She was admitted in the wards and initially managed as a case of acute coronary syndrome and community-acquired pneumonia. Pertinent physical examination findings included the following: BP 100/60 $\mathrm{mm} \mathrm{Hg}$, cardiac rate of 77 beats per minute (bpm), respiratory rate of 22 cycles per minute, no neck vein engorgement, bibasal rales, with a systolic ejection murmur heard over the lower left sternal border with no radiation to the carotids. The 12-lead electrocardiography showed sinus rhythm, normal axis, with left atrial abnormality, and LV hypertrophy with secondary ST-T wave abnormalities; chest radiography revealed enlarged heart with LV prominence with mild congestion. Two-dimensional transthoracic echocardiogram was done which showed markedly thickened walls of the left ventricle (Figs. 1 and 2) with a peak instantaneous continuous wave Doppler gradient across the basal LV segment of $194 \mathrm{~mm} \mathrm{Hg}$, and concentric LV remodeling with segmental wall motion abnormality with preserved systolic function and presence of SAM of the mitral valve with an LV ejection fraction of 57\% by Simpson's. Surgical myectomy was advised but she refused any surgical treatment. ASA was then the treatment option.

On the second hospital day, she underwent coronary angiogram which showed patent stent on the RCA with absence of a major septal perforator branch of the LAD artery (Figs. 3-5). Figure $3 \mathrm{~b}$ shows how a normal major septal perforator appears on angiogram as it originates from the proximal LAD. This was taken from another patient, a 52-year-old female [11]. Septal

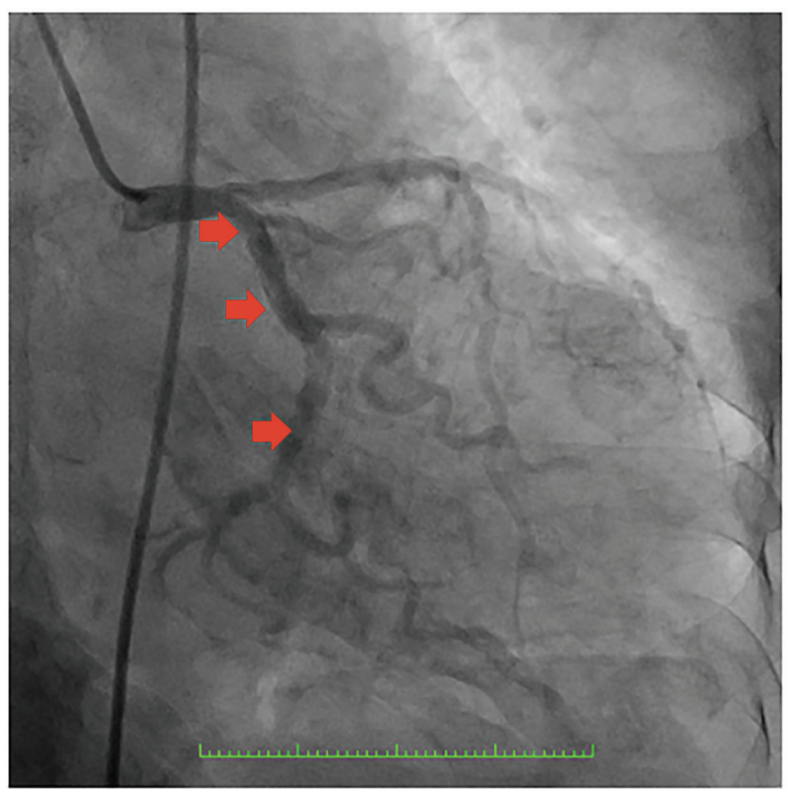

Figure 4. Coronary angiography showing the left circumflex coronary artery which was a good-sized vessel with no significant stenosis (arrows). 


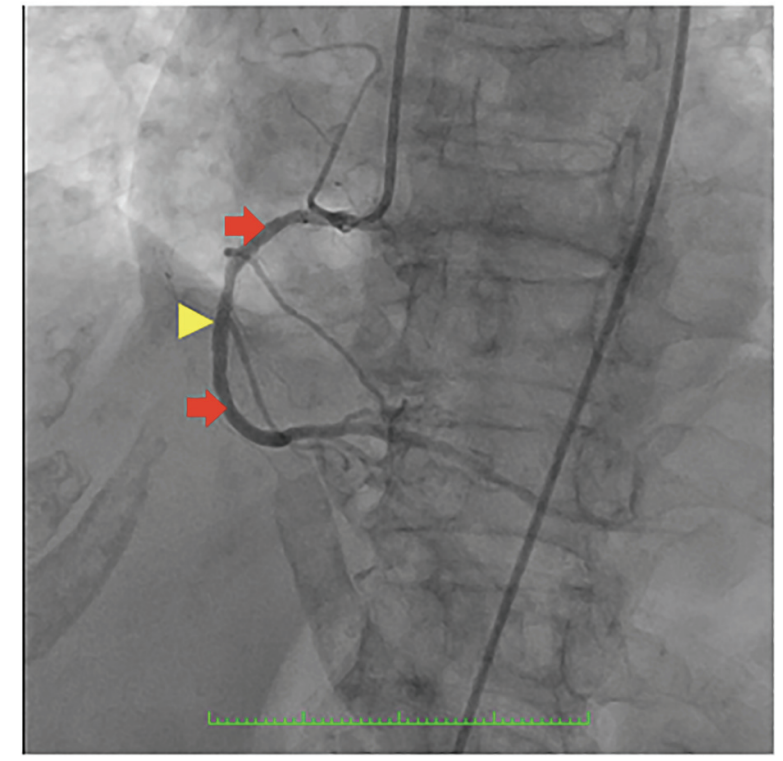

Figure 5. Coronary angiography showing the right coronary artery (RCA) (red arrows) which was a good-sized vessel with patent stent at the proximal to mid segment (yellow arrowhead) with good flow and with no significant stenosis.

ablation was deferred and she was referred to electrophysiology for evaluation. On the third day, electrophysiologic/ventricular tachycardia (VT) studies were done which revealed a sinus rhythm, with a cardiac rate of $77 \mathrm{bpm}$, and a maximum rate of $140 \mathrm{bpm}$, no significant sinus pause, atrioventricular blocks, intraventricular conduction delays; on isoproterenol infusion, she had atrial tachycardia and atrial fibrillation with rapid ventricular response and developed congestion and hypoxia. She was given with one shock of synchronized cardioversion, 150 $\mathrm{J}$ and was transferred to coronary care unit for close monitoring. Electrolytes were corrected accordingly. After she was stabilized, she was transferred to regular room and was scheduled for dual chamber (DDD) pacemaker implantation. After implantation of pacemaker, she remained stable and symptomfree. A repeat transthoracic echocardiogram was done which revealed significant decrease in peak instantaneous gradient basal LV segment from 197 to $37 \mathrm{~mm} \mathrm{Hg}$ (Fig. 6), the SAM of the mitral valve shown before was no longer appreciated, and there was overall improvement in wall motion. The presence of pacemaker lead in place was identified. She was discharged on the 10th hospital day stable and asymptomatic.

She had regular outpatient follow-up and up to 1 year post-pacemaker implantation, she remained asymptomatic.

\section{Discussion}

\section{Treatment strategies in $\mathrm{HCM}$}

The American College of Cardiology/European Society of Cardiology, and American Heart Association have recommended surgical septal myectomy as the preferred primary management option for patients with HCM with severe drugrefractory NYHA class III/IV symptoms and those with obstruction to LV outflow under basal conditions with physiologic exercise $(\geq 50 \mathrm{~mm} \mathrm{Hg})[2,3,10]$. The objective of surgery is the reduction in heart failure symptoms and improved quality of life, by virtue of relieving the SAM and outflow obstruction, and normalizing LV pressures. In patients who underwent surgery, $95 \%$ of patients experienced permanent eradication of the basal outflow gradient and $85 \%$ experienced symptom

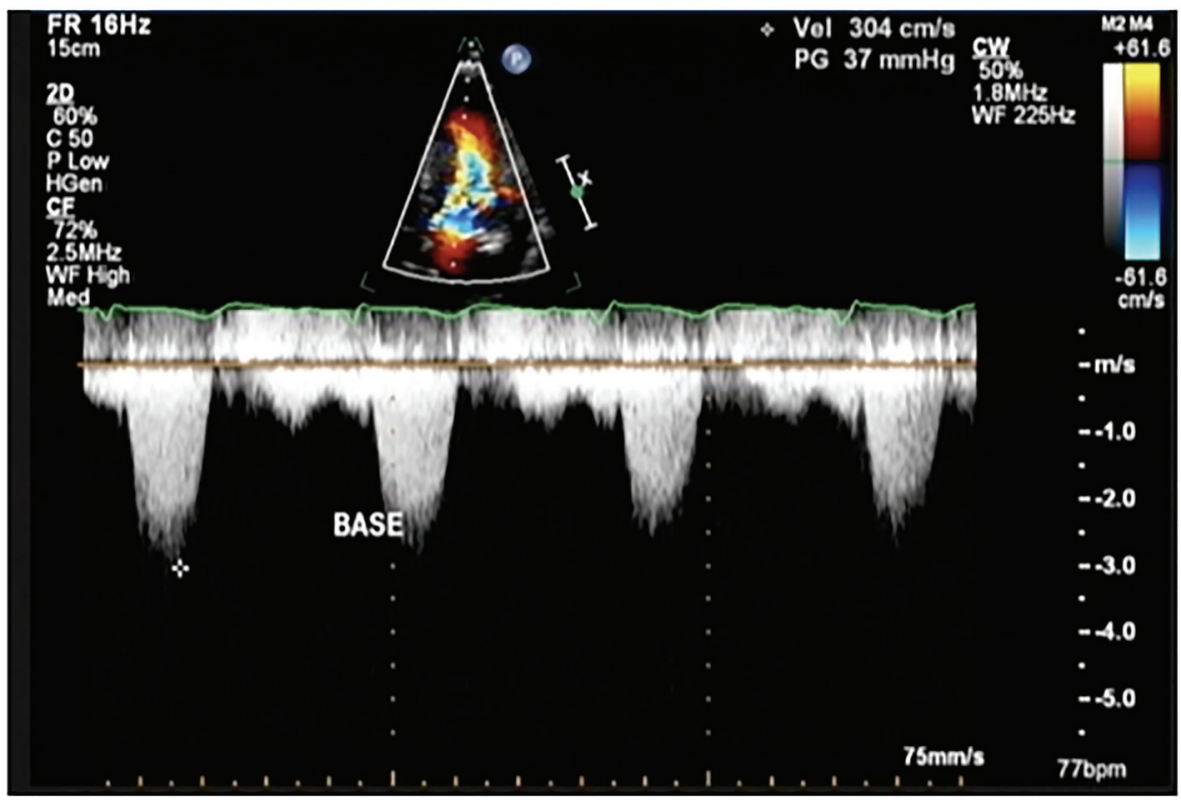

Figure 6. Color flow Doppler study of the patient showing a peak instantaneous gradient of $37 \mathrm{~mm} \mathrm{Hg}$ at post-implantation of dual chamber pacemaker (This was previously described as $194 \mathrm{~mm} \mathrm{Hg}$ before implantation pacemaker). 
relief during periods of up to 25 years $[2,3,10]$. Surgery is not recommended in patients who are asymptomatic or mildly symptomatic because of the lack of conclusive evidence that prophylactic relief of obstruction is advantageous. Its operative mortality has decreased steadily and is now less than $1 \%$ at selected myectomy centers [12].

ASA is an alternative to myectomy and involves injection of $1-3 \mathrm{~mL}$ of $95 \%$ alcohol into a major septal perforator coronary artery to create necrosis and a permanent transmural myocardial infarction localized to the proximal ventricular septum which leads to progressive thinning and restricted basal septal excursion, and reduction in LV outflow tract gradient. ASA is considered an alternative treatment modality to septal myectomy in the following settings: 1) patients whose symptoms limit daily activities (functional class III or more, or exercise-induced syncope) despite medical therapy or if medical therapy cannot be tolerated; 2) patients with a significant level of outflow obstruction (i.e. pressure drop $>50-60 \mathrm{~mm} \mathrm{Hg}$ with provocation by a Valsalva maneuver, bicycle stress, or postextrasystolic augmentation); and 3) patients with a suitable LV and coronary morphology, that is, those with a "classical", subaortic obstruction produced by the protruding septum and the "SAM" of the mitral valve and one or more septal perforator arteries that serve the septal area. Several published studies on ASA demonstrated immediate reductions in the mean resting LVOT gradient from 65 to $17 \mathrm{~mm} \mathrm{Hg}$ and the mean post-extrasystolic gradient from 125 to $53 \mathrm{~mm} \mathrm{Hg}$, with persistence of reduction even after 12 months of treatment $(16$ and $32 \mathrm{~mm} \mathrm{Hg}$, respectively) [13]. Meta-analyses have indicated no difference between septal ablation and myectomy in the medium-term incidence of sudden cardiac death or all-cause mortality [13].

Dual chamber pacemaker implantation was proposed as an alternative treatment for patients with severe symptomatic obstructive HCM. Although the exact mechanism of improvement with pacing remains unknown, the decrease in gradient may be caused by timing of septal contraction but may also reflect long-term remodeling. Although there was an initial enthusiasm for dual chamber pacing as a primary treatment for patients with obstructive HCM, subsequent RCTs demonstrated long-lasting beneficial results in only a small minority of patients $[14,15]$. The overall success rate in symptom relief and gradient reduction is significantly lower compared to those patients who undergo septal myectomy $[14,15]$. The mean residual gradient after septal myectomy is $10 \mathrm{~mm} \mathrm{Hg}$ compared with a $40-50 \mathrm{~mm} \mathrm{Hg}$ gradient after dual chamber pacing [14, 15]. Patients $>65$ years of age may be a subgroup who achieve the greatest benefit [14].

\section{Individualizing treatment options}

In our case, the patient was appraised for surgical myectomy. However, she refused surgery and opted for an alternative, less invasive option - ASA. Unfortunately, on coronary angiography, there was absence of major septal perforator coronary anatomy rendering her unsuitable for ASA. Consequently, an alternate option (dual chamber pacing) was offered with the goal of reducing the LV outflow tract gradient and improvement of symptoms. The absence of septal perforator branches of the LAD artery was described by Angelini et al in 1999 and classified under "other anomalies" with an incidence of $0.27 \%$ using 1950 coronary angiograms [16]. While the first septal perforator branch of the LAD artery is the typical channel for ASA, HCM patients with severe septal hypertrophy may rarely present with unusual septal perforator anatomy [17]. The absence of septal perforator coronary arteries in these patients renders them unsuitable for ASA [3].

\section{Outcome and follow-up}

She underwent the dual chamber pacemaker implantation as alternate to ASA and documented immediate significant reduction in the peak instantaneous gradient across the basal segment and obliteration of the SAM of the mitral valve. There was also noted improvement in overall systolic function. These hemodynamic changes were coupled with symptomatic improvement as the patient remained stable and asymptomatic after implantation. One year after implantation of dual chamber pacemaker, the patient had an outpatient follow-up and was reported to have absence of symptom recurrence since she was discharged.

\section{Conclusion}

We presented a case of HCM with angiographically absent major septal perforator branch of the LAD coronary artery treated with dual chamber pacemaker implantation to reduce the LV outflow gradient. To our knowledge, our case is not only the first reported angiographically absent first septal perforator coronary anatomy in the setting of HCM, but also the first description of dual chamber pacemaker implantation to relieve the LVOT obstruction.

Although the role of dual chamber pacing has become limited in HCM because surgical myectomy has set the standard for therapy and ASA has been accepted as suitable alternative, this modality remains essential and may still be considered as the treatment strategy of choice in a subset of patients who refuse (or high risk for) surgery and who are not suitable for ASA.

\section{Acknowledgments}

The authors would like to thank their colleagues, friends and family who in one way or another have contributed for the completion and improvement of this paper. This is dedicated to patients with hypertrophic cardiomyopathy who are unsuitable for surgery and septal ablation, as this report serves as a reminder that whenever encountered with a therapeutic dilemma, there will always be "another option".

\section{Conflicts of Interest}

The authors have no conflicts of interest to disclose. 


\section{References}

1. Mann DL, Zipes DP, Libby P, Bonow RO. Braunwald's heart disease: a textbook of cardiovascular medicine. 10th ed. Philadelphia: Elsevier; 2014. p.1574-1588.

2. Maron BJ, Maron MS. Hypertrophic cardiomyopathy. Lancet. 2013;381(9862):242-255.

3. Maron BJ, McKenna WJ, Danielson GK, Kappenberger LJ, Kuhn HJ, Seidman CE, Shah PM, et al. American College of Cardiology/European Society of Cardiology clinical expert consensus document on hypertrophic cardiomyopathy. A report of the American College of Cardiology Foundation Task Force on Clinical Expert Consensus Documents and the European Society of Cardiology Committee for Practice Guidelines. J Am Coll Cardiol. 2003;42(9):1687-1713.

4. ten Cate FJ, Soliman OI, Michels M, Theuns DA, de Jong PL, Geleijnse ML, Serruys PW. Long-term outcome of alcohol septal ablation in patients with obstructive hypertrophic cardiomyopathy: a word of caution. Circ Heart Fail. 2010;3(3):362-369.

5. Nishimura RA, Ommen SR. Septal reduction therapy for obstructive hypertrophic cardiomyopathy and sudden death: what statistics cannot tell you. Circ Cardiovasc Interv. 2010;3(2):91-93.

6. Valeti US, Nishimura RA, Holmes DR, Araoz PA, Glockner JF, Breen JF, Ommen SR, et al. Comparison of surgical septal myectomy and alcohol septal ablation with cardiac magnetic resonance imaging in patients with hypertrophic obstructive cardiomyopathy. J Am Coll Cardiol. 2007;49(3):350-357.

7. Fananapazir L, Epstein ND, Curiel RV, Panza JA, Tripodi $\mathrm{D}$, McAreavey D. Long-term results of dual-chamber (DDD) pacing in obstructive hypertrophic cardiomyopathy. Evidence for progressive symptomatic and hemodynamic improvement and reduction of left ventricular hypertrophy. Circulation. 1994;90(6):2731-2742.

8. Jeanrenaud X, Goy JJ, Kappenberger L. Effects of dualchamber pacing in hypertrophic obstructive cardiomyopathy. Lancet. 1992;339(8805):1318-1323.

9. McDonald K, McWilliams E, O'Keeffe B, Maurer B. Functional assessment of patients treated with permanent dual chamber pacing as a primary treatment for hyper- trophic cardiomyopathy. Eur Heart J. 1988;9(8):893-898.

10. Gersh BJ, Maron BJ, Bonow RO, Dearani JA, Fifer MA, Link MS, Naidu SS, et al. 2011 ACCF/AHA guideline for the diagnosis and treatment of hypertrophic cardiomyopathy: a report of the American College of Cardiology Foundation/American Heart Association Task Force on Practice Guidelines. Circulation. 2011;124(24):e783831.

11. Moreno-Martinez FL, Vega L, Fleites H, Ibargollin R, Gonzalez R, Lopez O. Dual left anterior descending coronary artery. Internet J Thorac Cardiovasc Surg. 2004 [cited 2015 Mar 25];7(1). Available from: URL:

12. Maron BJ. Controversies in cardiovascular medicine. Surgical myectomy remains the primary treatment option for severely symptomatic patients with obstructive hypertrophic cardiomyopathy. Circulation. 2007;116(2):196206; discussion 206.

13. Leonardi RA, Kransdorf EP, Simel DL, Wang A. Metaanalyses of septal reduction therapies for obstructive hypertrophic cardiomyopathy: comparative rates of overall mortality and sudden cardiac death after treatment. Circ Cardiovasc Interv. 2010;3(2):97-104.

14. Maron BJ, Nishimura RA, McKenna WJ, Rakowski H, Josephson ME, Kieval RS. Assessment of permanent dual-chamber pacing as a treatment for drug-refractory symptomatic patients with obstructive hypertrophic cardiomyopathy. A randomized, double-blind, crossover study (M-PATHY). Circulation. 1999;99(22):2927-2933.

15. Nishimura RA, Trusty JM, Hayes DL, Ilstrup DM, Larson DR, Hayes SN, Allison TG, et al. Dual-chamber pacing for hypertrophic cardiomyopathy: a randomized, doubleblind, crossover trial. J Am Coll Cardiol. 1997;29(2):435441.

16. Angelini P, Villason S, Chan AV Jr, Diez JG. Coronary artery anomalies: a comprehensive approach [online]. Philadelphia: Lippincott Williams \& Wilkins; 1999 [cited 2015 Mar 25]. Available from URL: http://digitalcommons.library.tmc.edu/cgi/viewcontent.cgi?article=1005 \& context=angelini.

17. Choi D, Dardano J, Naidu SS. Alcohol septal ablation through an anomalous right coronary septal perforator: first report and discussion. J Invasive Cardiol. 2009;21(6):e106-109. 